\title{
Median Nerve Compression by Gouty Tophi: A Case Report
}

\author{
Raffael Ia,b, Rajesh S \\ aDepartment of Orthopaedics \& Traumatology, UKMMC \\ ${ }^{\mathrm{D} D e p t}$ of Orthopaedics, Traumatology \& Rehabilitation, IIUM, Malaysia
}

\begin{abstract}
We describe a case of median nerve compression by gouty tophi. This is a rare condition and presented with loss of function with paraesthesia of both hands in addition to multiple tophis. Surgery was chosen for immediate relief, where a debulking of left flexor digitorum superficialis with decompression of carpal tunnel was done and resulted in improvement of symptoms but became complicated due to inadequate physiotherapy.
\end{abstract}

KEYWORDS: Carpal Tunnel Syndrome, Median Nerve Compression, Gouty tophi

\section{CASE REPORT}

Patient is a 50 year old right handed retired army officer, who currently works as a security guard. He presented to us with progressive discomfort and paraesthesia of both palms since 2 years ago with involvement of the radial 3 fingers. The symptoms worsened until 2 months ago where patient claimed loss of some fine motor of the thumb, index finger and middle fingers resulting in difficulty of daily activities such as buttoning clothes, tying shoelaces and utilizing utensils for eating with occasional episodes of dropping small items accidentally.

According to him, symptoms did not improve with splinting or change in hand position and the discomfort would cause some distress especially at night. In addition to these complaints, patient has also been taking NSAIDs prescribed to him by a private practitioner for recurrent exacerbation of gouty arthritis. However, he has never had his serum uric acid levels checked nor is he under any diet restrictions.

General examination revealed a comfortable middle aged gentleman with multiple painless gouty tophi over both $1^{\text {st }}$ metatarsal heads, soles of the feet,

\section{Corresponding author:}

Dr. Raffael bin Ismail

Department of Orthopaedics, Traumatology and Rehabilitation,

Kulliyyah (Faculty) of Medicine, International Islamic University Malaysia, Jalan Hospital, 25150 Kuantan, Pahang, Malaysia

Tel: $\quad+60192105637$

Fax: $\quad+6095144451$

Email: raffaelismail@gmail.com.my lateral malleoli and the distal interphalangeal joint of the right thumb.

Examination of the hands revealed muscle atrophy over both thenar regions and weakness of the thumb abduction. Numbness was prominent for the volar aspect of the thumb, index and middle finger. Tinel's sign was present upon tapping at the proximal end of the transverse flexor carpal ligament and Phalen's sign was present. Symptoms were similar in both hands but were more pronounced at the left hand. There were no palpable lesions on both wrists. There was no restriction of wrist joint movement.

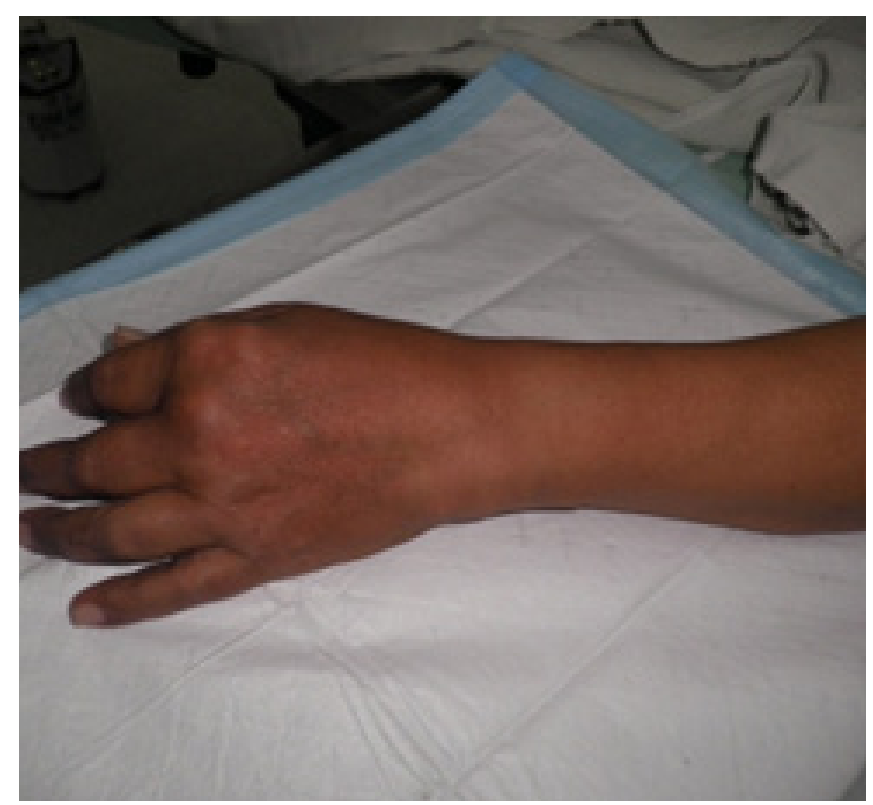

Figure I. Wasting of the $1^{\text {st }}$ lumbrical with tophi deposition at the index and middle finger metacarpophalangeal joint 


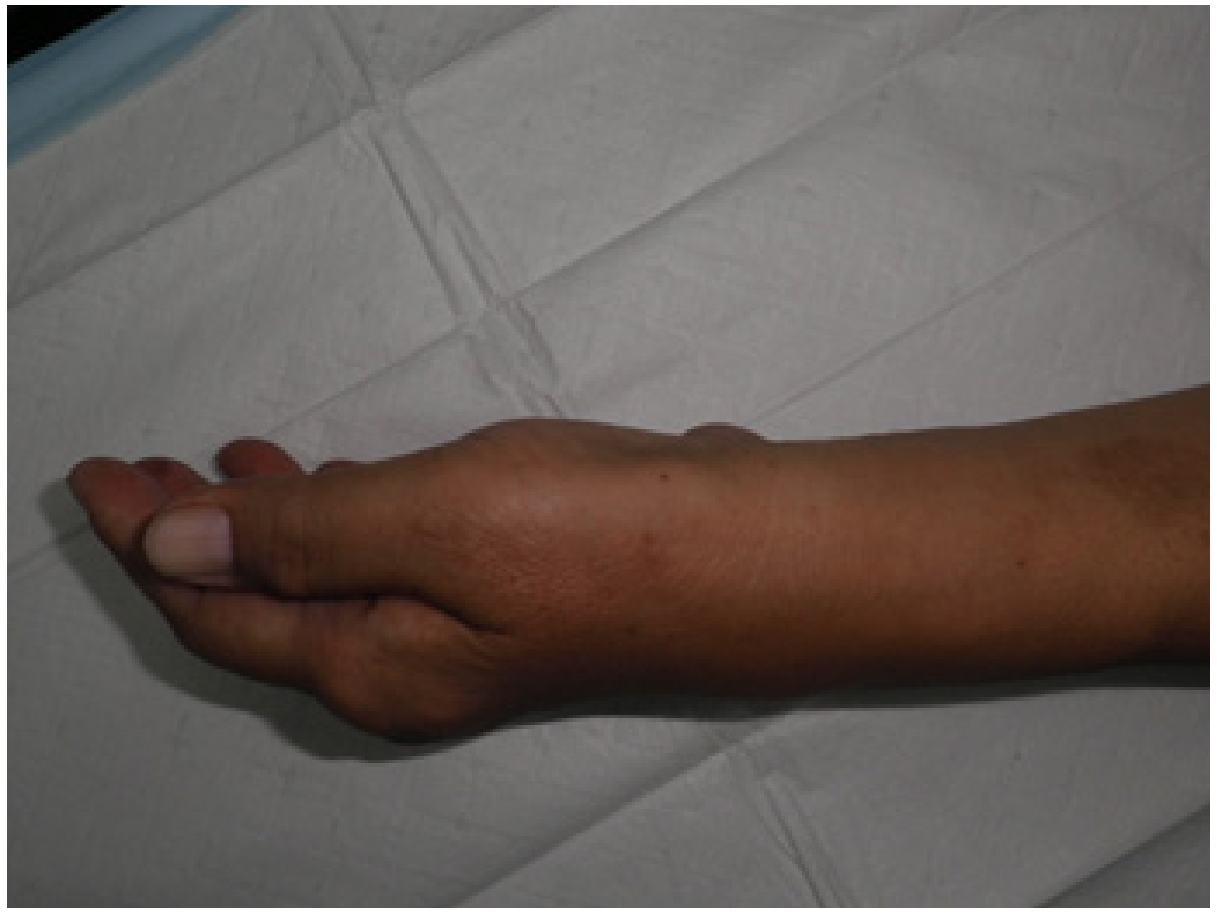

Figure II. Wasting of the thenar muscles secondary to median nerve neuropathy

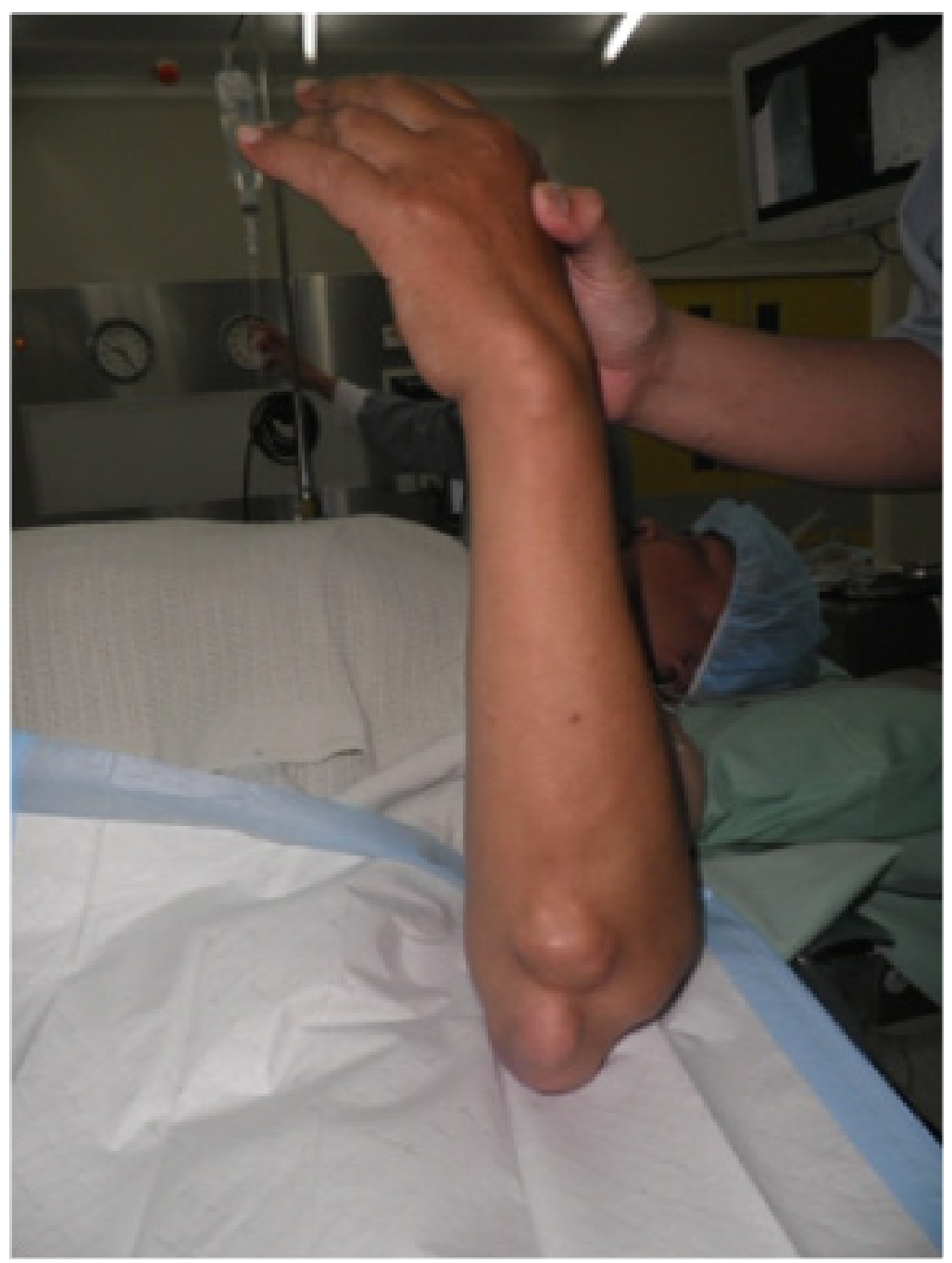

Figure III. Multiple tophi at the proximal and distal ulna. Lesions were abundant, painless and not inflamed 


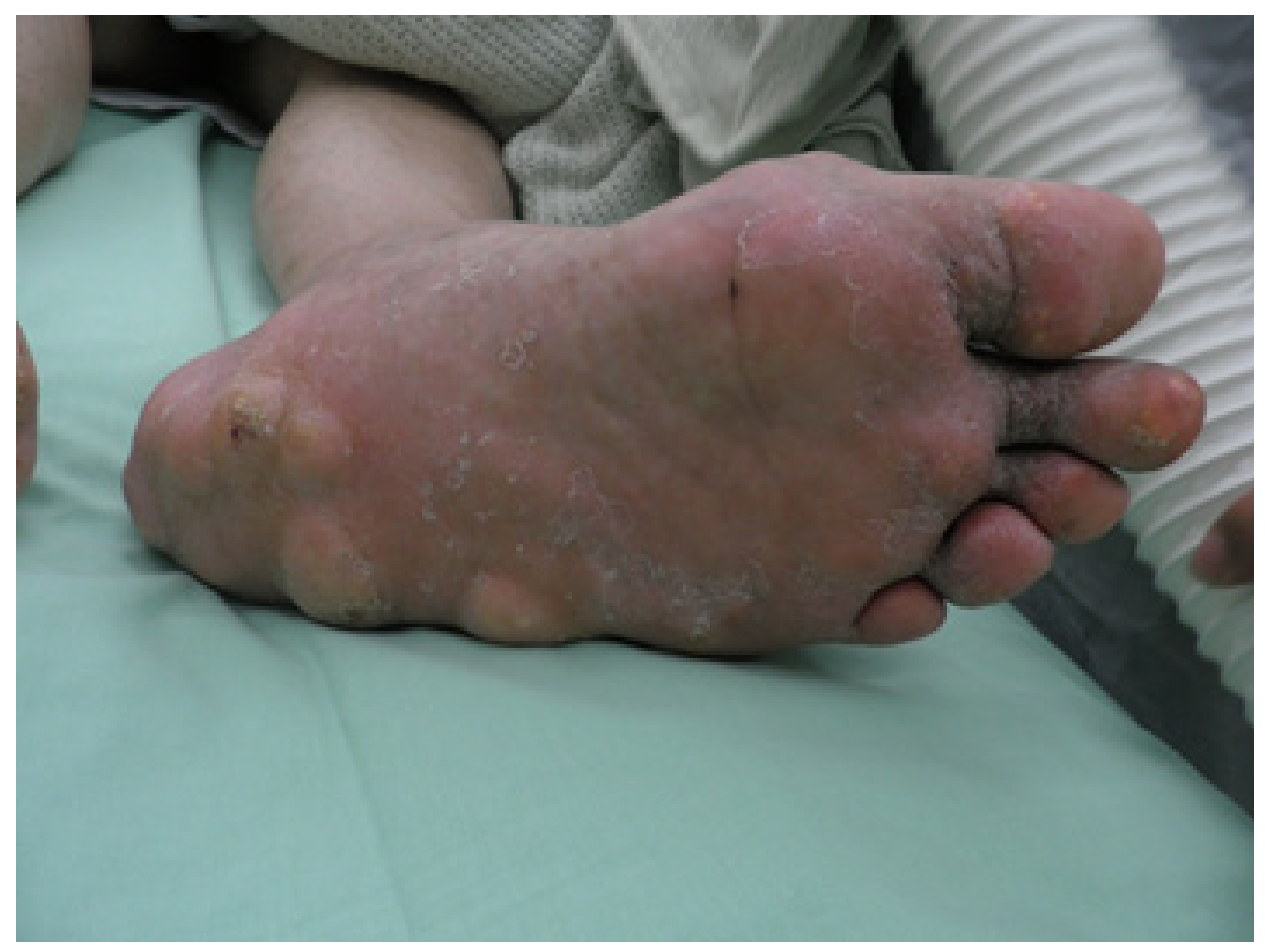

Figure IV. Multiple tophi at the left foot. Tophi also found on the dorsum and contralateral foot

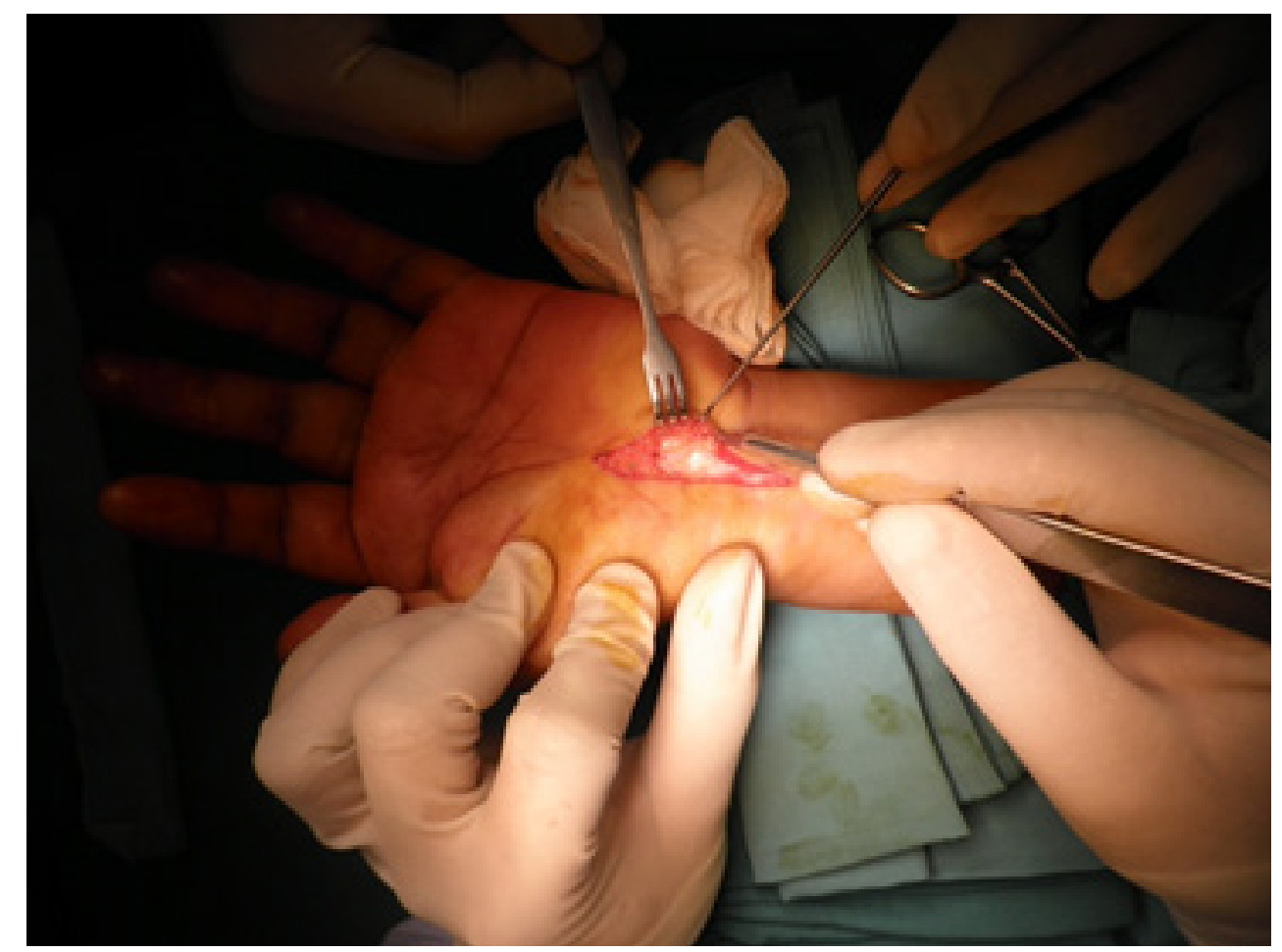

Figure V. Tophi deposition noted at the tendon of the flexor digitorum superficialis 
Laboratory findings showed elevated levels of serum uric acid on the day of admission at $603 \mu \mathrm{mol} / \mathrm{L}$. Other hematological investigations such as blood count and acute phase reactants were performed but were unremarkable.

Upon several consultation and discussion with the attending surgeon patient was explained that his symptoms was due to nerve compression. It was also explained that in view of multiple tophi formation, it could be possible that his symptoms could be contributed to direct compression by adjacent tophi.

After considering his dysfunction, patient agreed to have an open transverse flexor carpal ligament resection on his left wrist under general anaesthesia. Intraoperatively, the median nerve was flattened just proximal to the transverse carpal ligament to a point $3 \mathrm{~cm}$ proximal to it. It was also noted that tophi was seen encroaching the flexor digitorum superficialis along the point of the compression which was debulked. Exploration of the carpal tunnel and immediate proximity revealed no other depositions. Nevertheless, the transverse carpal ligament was resected to anticipate reactive inflammation which may result in nerve compression post-surgery.

Postoperatively, patient recovered well from surgery and upon follow up at one month paraesthesia had reduced to a point where patient could feel sharp pin pricks on previously insensate regions of the hands. However, it was noted that the overlying skin was warm and erythematous indicating an inflammation reaction after the first weeks of follow up with a reduction in finger range of movement despite attending several physiotherapy sessions.

\section{DISCUSSION}

Gout is a disorder of purine metabolism which predisposes to hyperuricaemia, leading to monosodium urate crystal depositions in joints. In addition to joints, uric acid crystals are reported to deposit at soft tissues such as tendons median nerve, bursae and intrinsic muscles. ${ }^{1,2}$ Studies also show these formations were seen at the base of carpal tunnel, carpal bones, radiocarpal joint and at extensor tendon sheaths. ${ }^{3}$ Hart et al disclosed occurrences of median nerve compression due to gouty tophi formation. ${ }^{4,5}$

The flexor carpal ligament roofs a rigid cannular pathway established by carpal bones walls on three sides. ${ }^{6}$ The tunnel contains the flexor pollicis longus encapsulated by the radial bursa and the ulnar bursa with its invested flexor digitorum superficialis and profundus tendon and the median nerve with its artery. Therefore, any increase in the contents of this tunnel causes an inward increase in pressure due to the rigidity of the structure causing median nerve compression. Even more so in cases of carpal tunnel syndrome caused by tophaceous gout as a synergistic insult on the median nerve occurs attributed to both direct tophi compression and associated tenosynovitis.

Compression neuropathy itself is a common nerve injury in the population, where carpal tunnel syndrome alone has prevalence of $8 \% .^{7}$ Of this value, an even smaller group has been identified to have median nerve compression due to gouty tophi and misdiagnosed as carpal tunnel syndrome. Rich et al performed 2,649 carpal tunnel releases and only 15 hands $(0.6 \%)$ had with gout ${ }^{8}$. Whereas, Patil and Chopra in 2007 found that despite a span of 25 year period and 316 patients, only 3 had tophaceous gout changes. ${ }^{8}$ These data show that a high level of suspicion may help in identifying the cause of the nerve compression despite a low prevalence rate.

Patients presenting with carpal tunnel syndrome have multiple features, both seen as sensory and motor deficit resulting from the compression of the median nerve. The pathophysiology is initiated by a reduction in blood flow of the epineural. This occurs from a compression of 20 to $30 \mathrm{mmHg} .{ }^{9}$ Compression eventually will lead to edema of the epineurium and endoneurium. Ongoing or an increment in pressure beyond 8 hours would result in increased endoneural fluid pressure up to fourfold thus resulting in impedance of axonal transport. ${ }^{10}$ In this patient, the insult lasted for more than two years and being a combination of direct compression of tophi and surrounding edema, any rest or postural change did little to alleviate the symptoms. With this, the cycle of disrupted epineural blood flow and edema will result in a vicious cycle. This is especially so as the edema is unable to pass through the perineurium and with its higher tensile strength, produces a "compartment syndrome" of the nerve by simulating a diffusion barrier. ${ }^{10}$

In this patient open carpal release was done in view of the nerve compression symptoms. However, instead of opting for endoscopic or minimal incision, a larger incision was chosen in view of the relevant history of neuropathy in addition to multiple gouty tophi over the peripheries. Several cases reported have mentioned additional features of abnormal hand function such as loss of finger flexion, stiffness and flexion contracture ${ }^{8}$ secondary to tophi deposition. Patients in these reports were reported to have said abnormalities in addition to median nerve neuropathy. Furthermore, patients were also known to have multiple joint pains with or without prominent tophi formation. In this patient however, there were no abnormal hand functions which were not related to median nerve compression preoperatively.

As mentioned previously, pharmacological therapy has been reported by Fujimoto et al. in 1968 to be effective in the treatment of median neuritis secondary to gout and remains a valid choice as it results in resolution of tophi, addressing the primary factor. However, this choice requires patient's compliance to their medication and symptomatic 
relief is not instantaneous. This results in a majority of patients opting for surgical debulking for a faster and immediate relief, in addition to prevent permanent damage and possible loss of function. Also important is postoperative care and rehabilitation, as inflammation reaction combined with a less than aggressive physiotherapy may result in serious pitfalls in the management of these patients amounting to a loss in hand function.

\section{REFERENCES}

1. Rich JT, Bush DC, Lincoski CJ, et al. Carpal tunnel syndrome due to tophaceous gout. Orthopedics 2004; 27:862-3.

2. Patil VS, Chopra A. Watch out for 'pins and needles' in hands-it may be a case of gout. Clin Rheumatol 2007; 26:2185-7.

3. Hart AM, Wiberg M. Nerve compression or mechanical neuropathy: neuropathology. Current Orthopaedics 2001; 15:245-248.

4. Chen CK, Chung CB, Yeh L, et al. Carpal Tunnel Syndrome Caused by Tophaceous Gout: CT and MR Imaging Features in 20 Patients. AJR Am J Roentgenol 2000; 175:655-9.

5. Robbins $\mathrm{H}$. Anatomical study of the median nerve in the carpal tunnel and etiologies of the carpal tunnel syndrome. J Bone Joint Surg Am 1963; 45:953-66.

6. Arle JE, Zager EL. Surgical treatment of common entrapments neuropathies in the upper limbs. Muscle Nerve 2000; 23:1160-74.

7. Patil VS, Chopra A. Watch out for 'pins and needles' in hands-it may be a case of gout. Clin Rheumatol 2007; 26:2185-7.

8. Sunderland $\mathrm{S}$. The nerve lesion in the carpal tunnel syndrome. J Neurol Neurosurg Psychiatry 1976; 39:615-26.

9. Gelberman RH, Rydevik BL, Pess GM, et al: Carpal tunnel syndrome: a scientific basis for clinical care. Orthop Clin North Am 1988; 19:115-24.

10. Lundborg G, Myers R, Powell H. Nerve compres sion injury and increased endoneurial fluid pressure a "miniature compartment syndrome." J Neurol Neurosurg Psychiatry 1983; 46:1119-24. 